\title{
Diagnosis of Brain Cancer Using Radial Basis Function Neural Network with Singular Value Decomposition Method
}

\author{
Agus Maman Abadi, Dhoriva Urwatul Wustqa, and Nurhayadi
}

\begin{abstract}
Brain cancer is one of the most dangerous cancers that can attack anyone, so early detection needs to be done so that brain cancer can be treated quickly. The purpose of this study is to develop a new procedure of modeling radial basis function neural network (RBFNN) using singular value decomposition (SVD) method and to apply the procedure to diagnose brain cancer. This study uses 114 brain Magnetic Resonance Images (MRI). The RBFNN model is constructed by using steps as follows; image preprocessing, image extracting using Gray Level Co-Occurrence Matrix (GLCM), determining of parameters of radial basis function and determining of weights of neural network. The input variables are 14 features of image extractions and the output variable is a classification of brain cancer. Before learning process, the input data is normalized. The modeling is done by using K-means clustering method where the activation function in the hidden layer is Gaussian function and by determining the optimum weights of the model using SVD method. The best RBFNN model is 14 input neurons, 10 hidden layer neurons, and 1 output neuron. The results show that the sensitivity, specificity, and accuracy of RBFNN diagnoses with backpropagation equal to those of RBFNN with SVD. However, the RBFNN-SVD delivers an advantage in the running speed of the program.
\end{abstract}

Index Terms-Radial basis function neural network, diagnosis brain cancer, singular value decomposition.

\section{INTRODUCTION}

Brain cancer is a dangerous disease and can be fatal to someone. In 2012, brain cancer cases in Indonesia are 1.9 per 100,000 population, while the brain mortality rate is 1.3 per 100,000 inhabitants [1]. This fact shows the level of brain cancer patients in Indonesia is high. Therefore, early diagnosis is needed to prevent the death caused by brain cancer. According to the Ministry of Health of the Republic of Indonesia [2], there are several steps in diagnosing brain cancer (1) physical examination; (2) laboratory examination; and (3) radiological examination. Standard radiological examinations are CT Scan and MRI which produce the digital images. The radiologists interpret the MRI images by

Manuscript received October 20, 2018; revised April 26, 2019. This work was supported in part by the Directorate of Research and Community Service, Ministry of Research, Technology, and Higher Education of the Republic of Indonesia under grant No. 01/Penelitian/ PDUPT/ UN.34.21/2018.

Agus Maman Abadi and Dhoriva Urwatul Wutsqa are with Mathematics Departnment, Yogyakarta State University, Karangmalang Yogyakarta Indonesia, 55281 (e-mail: agusmaman@uny.ac.id, dhoriva_uw@uny.ac.id).

Nurhayadi is with Mathematics Education Department, Tadulako University, Sulawesi Tengah, Indonesia (e-mail: nurhayadi@gmail.com). observation and analysis them. Then, they consult the results of the analysis to the doctor. The diagnosis using MRI image can only categorize the brain as normal or abnormal.

Regarding the important of the early diagnosis of brain cancer, many researchers are tempted doing research in this field. Several methods have been applied to classify brain cancer, such as artificial neural networks based on brain MRI data [3], [4], and backpropagation network techniques (BPNN) and principal component analysis (PCA) [5], probabilistic neural network method [6], [7]. Several researches have proposed the combining several intelligent system methods, those are adaptive neuro-fuzzy inference system (ANFIS), neural Elman network (Elman NN), nonlinier autoregressive with exogenous neural network (NARXNN), and feedforward NN [8] and artificial neural network based on MRI image segmentation data using fuzzy c-means and bounding Box method [9]. Studies to diagnose brain cancers have also been done using support vector machine (SVM) and multi-SVM [10], adaptive neuro-fuzzy inference system [11].

Recently, research on brain cancer diagnosis still become interesting area to develop the method in order to improve accuracy of the diagnosis. One of the methods that can be used as diagnosis tool is the radial basis function neural network (RBFNN). The RBFNN is suitable for large training data, provide a good generalization. The RBFNN has simple network structure, since it has only one hidden layer. This property leads to speed up the learning process. Study related to the diagnosis of brain cancer by applying RBFNN has been performed using CT scan data of normal and abnormal brain [12]. The other study uses principle component analysis based on electroencephalograph (EEG) digital signals [13] and set those values as the inputs of RBFNN model.

The RBFNN model has been applied to diagnose various types of diseases. The classification of breast cancer using improved F-score with support vector machine and RBF network has been done [14]. The result shows that the improved F-score and support vector machines method gives the better accuracy than improved F-score and RBF network method. The study of cancer prediction based on gene expression data using deep learning has been done [15]. The modified neural network has been applied to diagnosis of epilepsy based on EEG signals and the result shows that the ensemble TPunit neural networks method gives the better accuracy than neural network method for diagnosis of epilepsy [16]. The RBFNN model has been applied for detection of lung cancer based on radiograph images [17].

In the RBFNN modeling, the weights of output target can be determined by back propagation or global ridge regression methods. However, these methods require a long iteration to get the solution, while singular value decomposition method does not require iteration and is more efficient for 
determining the weights of output target. This study aims to diagnose brain cancer based on MRI image data using the combination of radial basis function neural network (RBFNN) and singular value decomposition method.

\section{RADIAL BASIS FunCTION NEURAL NETWORK}

The RBFNN is one of the neural network model with a three-layer, namely an input layer, a single hidden layer, and an output layer. The performance of RBFNN depends on the choice of exactly three important parameters (cluster center, distance and weight). Each input variable is assigned to the neuron in the input layer and enters directly to the hidden layer without weight. In the hidden layer, the RBFNN performs a nonlinear transformation of the data from the input layer using the basic radial function before it is linearly processed at the output layer. The architecture of the RBFNN is shown in Fig. 1.

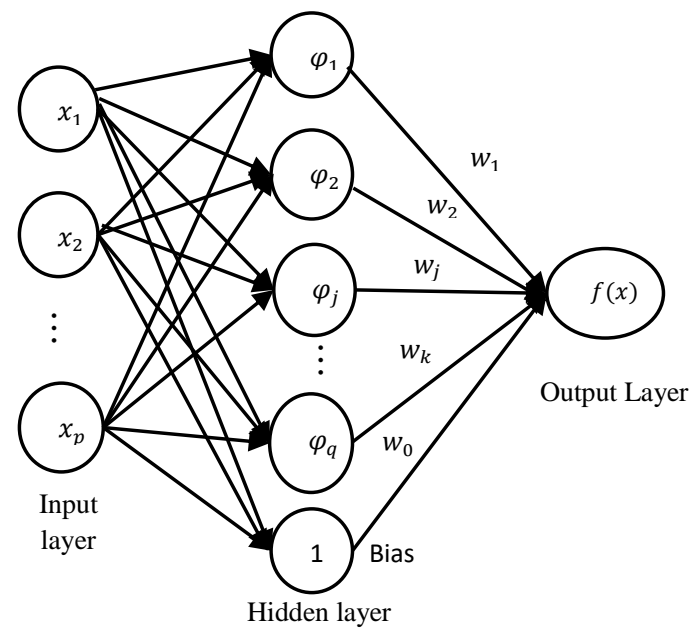

Fig. 1. The architecture of RBFNN.

In Fig. $1,\left(x_{i}, i=1,2, \ldots p\right)$ are input neurons, $\left(\varphi_{j}, j=\right.$ $1,2, \ldots, q)$ are hidden neurons, and $f(x)$ is output neuron. The weights between the hidden layer and the output layer are denoted as $w_{j}$ and the bias $w_{0}$ is added to the hidden layer. The activation function $\varphi_{j}(x)$ is determined by the mean cluster and width cluster. The RBFNN model is characterized by the activation function, which are included in the class of radial basis function. There are several activation functions in RBFNN namely Gaussian function, multi quadratic inverse and Cauchy functions [18]. In this research, we use Gaussian activation function

$$
\varphi(x)=\exp \left(-\frac{(x-c)^{2}}{r^{2}}\right)
$$

where $c$ is the mean of cluster, $r$ is maximum distance of each object to the mean of cluster. The output $y$ of the RBFNN model is the linear combination of the weights $w_{j}$ included $w_{0}$ and the activation function $\varphi_{j}(x)$

$$
\begin{gathered}
f(\boldsymbol{x})=\sum_{j=1}^{q} w_{j} \varphi_{j}(\boldsymbol{x})+w_{0} \\
=\sum_{j=1}^{q} w_{j} \exp \left(-\sum_{i=1}^{p} \frac{\left(x_{i}-c_{j i}\right)^{2}}{r_{j i}{ }^{2}}\right)+w_{0}
\end{gathered}
$$

The learning process in RBFNN includes unsupervised learning to ascertain the number of hidden neurons as well as to obtain the parameter values of the radial basis function, and supervised learning to obtain the weights between the hidden layer and output layer. The number of neurons in the hidden layer equals to the number of basis functions. The unsupervised learning is performed using the K-means clustering method. It is commonly used in RBFNN modeling, whose placement of the objects in each cluster based on the Euclidian distance. The Euclidian distance between two points $P\left(x_{1}, x_{2}, \ldots, x_{p}\right)$ and $Q\left(y_{1}, y_{2}, \ldots, y_{p}\right)$ is expressed as

$$
d(P, Q)=\sqrt{\left(x_{1}-y_{1}\right)^{2}+\ldots+\left(x_{p}-y_{p}\right)^{2}}
$$

The following steps are the K-means clustering algorithm [19].

1) Partition the data into $K$ cluster

2) Place any objects to the nearest cluster.

3) Recalculate the center of the clusters that receive new data and the clusters that lose data.

4) Repeat step (1) to (3) until the new centers equal to the old ones.

Futhermore, it will be determined the weight $\omega_{j}$ in (2) minimized the equation (3).

$$
S S E=\sum_{k=1}^{n}\left(y_{k}-\hat{y}_{k}\right)^{2}
$$

where $n$ is the number of training data, $\hat{y}_{k}$ is the output of the model for $k^{\text {th }}$ observation, $y_{k}$ is the target for $k^{\text {th }}$ observation.

Then, we apply (2) and (3) for all training data to get (4).

$$
\boldsymbol{y}=\left[\begin{array}{c}
y_{1} \\
y_{2} \\
\vdots \\
y_{n}
\end{array}\right]=\boldsymbol{\varphi} \widehat{\boldsymbol{w}}
$$

where

$$
\boldsymbol{\varphi}=\left[\begin{array}{ccccc}
\varphi_{1}[\mu(x)]_{1} & \varphi_{2}[\mu(x)]_{1} & \cdots & \varphi_{q}[\mu(x)]_{1} & 1 \\
\varphi_{1}[\mu(x)]_{2} & \varphi_{2}[\mu(x)]_{2} & \cdots & \varphi_{q}[\mu(x)]_{2} & 1 \\
\vdots & \vdots & \ddots & \vdots & 1 \\
\varphi_{1}[\mu(x)]_{n} & \varphi_{2}[\mu(x)]_{n} & \cdots & \varphi_{q}[\mu(x)]_{n} & 1
\end{array}\right]
$$

and $\widehat{\boldsymbol{w}}$ is a vector of weights $\widehat{w}_{j}$.

\section{Singular VALUE DeCOMPOSITION}

In this section, we will introduce singular value decomposition of matrix and its properties referred from Scheick [20]. Any $m \times n$ matrix $A$ can be expressed as

$$
A=U S V^{T}
$$

where $U$ and $V$ are orthogonal matrices of dimensions $m \times m$, $n \times n$ respectively and $S$ is $m \times n$ matrix whose entries are 0 except $s_{i i}=\sigma_{i} \quad i=1,2, \ldots, r$ with $\sigma_{1} \geq \sigma_{2} \geq \ldots \geq \sigma_{r}>0$, $r \leq \min (m, n)$. Equation (6) is called a singular value decomposition (SVD) of $A$ and the numbers $\sigma_{i}$ are called singular values of matrix $A$. If $U_{i}, V_{i}$ are columns of $U$ and $V$, respectively, then we can conclude as follows. 
1. $A=U\left(\begin{array}{ll}\Sigma & 0 \\ 0 & 0\end{array}\right) V^{T}$ with $\sum=\operatorname{diag}\left(\sigma_{1}, \sigma_{2}, \ldots, \sigma_{r}\right)$.

2. The matrices $A^{T} A$ and $A A^{T}$ are symmetric matrices and the coloumns of $U$ and $V$ are eigenvectors of $A A^{T}$ and $A^{T} A$, respectively.

3. The values $\sigma_{i}^{2}, i=1,2, \ldots, r$, are nonzero eigenvalues of $A^{T} A$ corresponding to eigenvectors $V_{i}$ and $V_{r+1}, \ldots, V_{n}$ are eigenvectors corresponding to zero eigenvalue.

4. The values $\sigma_{i}^{2}, i=1,2, \ldots, r$, are nonzero eigenvalues of $A A^{T}$ corresponding to eigenvectors $U_{i}$ and $U_{r+1}, \ldots, U_{m}$ are eigenvectors corresponding to zero eigenvalue.

5. $A V_{i}=\sigma_{i} U_{i}$ for $i=1,2, \ldots, r$ and $A V_{i}=0$ for $i>r$.

6. $A^{T} U_{i}=\sigma_{i} V_{i}$ for $i=1,2, \ldots, r$ and $A^{T} U_{i}=0$ for $i>r$.

Furthermore, the decomposition matrix $A$ in (6) can be written as

$$
A=\sum_{i=1}^{r} \sigma_{i} U_{i} V_{i}^{T}
$$

The SVD of matrix $A$ can be applied to solve the linear system $A x=d$. If $A$ is $n \times n$ invertible matrix, then $r=n$ and all $\sigma_{i}>0$. Hence $x=A^{-1} d=\sum_{i=1}^{n} \sigma_{i}^{-1}<d, U_{i}>V_{i}$ is the solution of the linear system where $<,>$ is standard inner product in $R^{n}$. If $A$ is singular matrix of arbitrary dimension, then the solution of $A x=d$ is

$$
x^{+}=\sum_{i=1}^{r} \sigma_{i}^{-1}<d, U_{i}>V_{i}
$$

Furthermore $\min \left\{\|A x-d\|: x \in F^{n}\right\}=\left\|A x^{+}-d\right\|$. It means that the optimal solution for the linear system $A x=d$ is matrix vector (8).

\section{DiagnOSTIC ACCURACY}

The diagnostic accuracy can be measured by sensitivity, specificity and accuracy. There are several commonly used terms along with descriptions of sensitivity, specificity and accuracy. Those are TP = True Positive, FP = False Positive, $\mathrm{TN}=$ True Negative, and FN = False Negative. The TP is the number of patients with the disease who is diagnosed appropriately, the $\mathrm{FN}$ is the the number of patients with the disease who is diagnosed inappropriately. Similiarly, the TN is the number of patients without the disease who is diagnosed appropriately, the FP is the the number of patients without the disease who is diagnosed inappropriately. The sensitivity refers to the ability of the test to identify patients with the disease appropriately [21], and it can be written as

$$
\text { Sensitivity }=\frac{T P}{T P+F N}
$$

The specificity refers to the ability of the test to identify patients without disease appropriately, and it can be written as

$$
\text { Specificity }=\frac{T N}{T N+F P}
$$

The accuracy is the ability to identify both positive and negative results appropriately, and it can be written as

$$
\text { Accuracy }=\frac{T N+T P}{T N+T P+F N+F P}
$$

\section{FEATURE EXTRACTION}

Texture is an important characteristic for identifying an object of image. Textural feature on spatial gray image is commonly used in classifying images. Gray Level Co-occurrence Matrix (GLCM) is one of extraction methods to to get textural feature of the image. It has capability to give detail information of image pattern [22]. The GLCM is included in statistical second order extracting method. GLCM is a matrix that stores the frequency value of the brightness differences between one pixel and other neigborhod pixel.

The use of GLCM is first introduced by [23] to extract some texture features. Several researches have used the GLCM method to extract the images [24]-[26]. The parameters resulted from GLCM extraction process in this research are 14 features involving energy, contrast, correlation, sum of squares, inverse difference moment, sum average, sum variance, sum entropy, entropy, difference variance, difference entropy, maximum probability, homogeneity, and dissimilarity [22], [23], [27], [28]. The mathematical formula of those features are as follows :

1. Energy $=\sum_{i=1}^{N_{g}} \sum_{j=1}^{N_{g}}(i j) P_{i j}^{2}$

2. Contrast $=\sum_{i=1}^{N_{g}} \sum_{j=1}^{N_{g}}(i-j)^{2} P_{i j}$

3. Correlation $=\frac{\sum_{i=1}^{N g} \sum_{j=1}^{N g}(i j) P_{i j}-\mu_{x} \mu_{y}}{\sigma_{x} \sigma_{y}}$

4. sum of squares $=\sum_{i=1}^{N_{g}} \sum_{j=1}^{N_{g}}(i-\mu)^{2} P(i, j)$

5. Inverse Difference Moment $=\sum_{i=1}^{N_{g}} \sum_{j=1}^{N_{g}} \frac{P_{i j}}{1+(i-j)^{2}}$

6. sum average $=\sum_{k=2}^{2 N_{g}} k P_{x+y}(k)$

7. sum variance $=\sum_{i=2}^{2 N_{g}}(i-S E)^{2} P_{x+y}(k)$

8. sum entropy $=-\sum_{k=2}^{2 N_{g}} P_{x+y}(k) \log \left\{P_{x+y}(k)\right\}$

9. Entropy $=-\sum_{i=1}^{N_{g}} \sum_{l=1}^{N_{g}} P_{i j} \log \left(P_{i j}\right)$

10. Difference variance $(D V)=$ variansi of $P_{x+y}(k)$

11. difference entropy $=-\sum_{j=0}^{N_{g}-1} P_{x+y}(k) \log \left\{P_{x+y}(k)\right\}$

12. maximum probability $=\max _{i, j}\{P(i, j)\}$

13. Homogenity $=\sum_{i=1}^{N_{g}} \sum_{j=1}^{N_{g}} \frac{P_{i j}}{1+|i-j|}$

14. Dissimilarity $=\sum_{i=0}^{N_{g}-1} \sum_{j=0}^{N_{g}-1} P(i, j)|i-j|$

where $i, j$ are the spatial coordinate of probability function $P_{i j}$, that is an entry ith row and jth colomn on GLCM. $N_{g}$ is the number of gray level of the image, and 


$$
\begin{aligned}
& \mu_{x}=\sum_{i=1}^{N_{g}} \sum_{j=1}^{N_{g}} i P(i, j) \\
& \mu_{y}=\sum_{i=1}^{N_{g}} \sum_{j=1}^{N_{g}} j P(i, j) \\
& \sigma_{x}=\sum_{i=1}^{N_{g}} \sum_{j=1}^{N_{g}}\left(i-\mu_{x}\right)^{2} P(i, j) \\
& \sigma_{y}=\sum_{i=1}^{N_{g}} \sum_{j=1}^{N_{g}}\left(j-\mu_{y}\right)^{2} P(i, j) \\
& P_{x+y}(k)=\sum_{i=1}^{N_{g}} \sum_{j=1}^{N_{g}} P(i, j) ; i+j=k ; \\
& k=2,3, \ldots, 2 N_{g}
\end{aligned}
$$

\section{The Proposed Method}

The data used in this research are the secondary data of Magnetic Resonance Image (MRI) of brain taken from the hospital of Panti Rapih Yogyakarta, Indonesia. The used data are 114 brain MRI where 57 data are normal images and 57 data are cancer images. In this research, the data are analyzed using RBFNN with singular value decomposition method.

The procedure to diagnose brain cancer using RBFNN-SVD model is shown in Fig. 2.

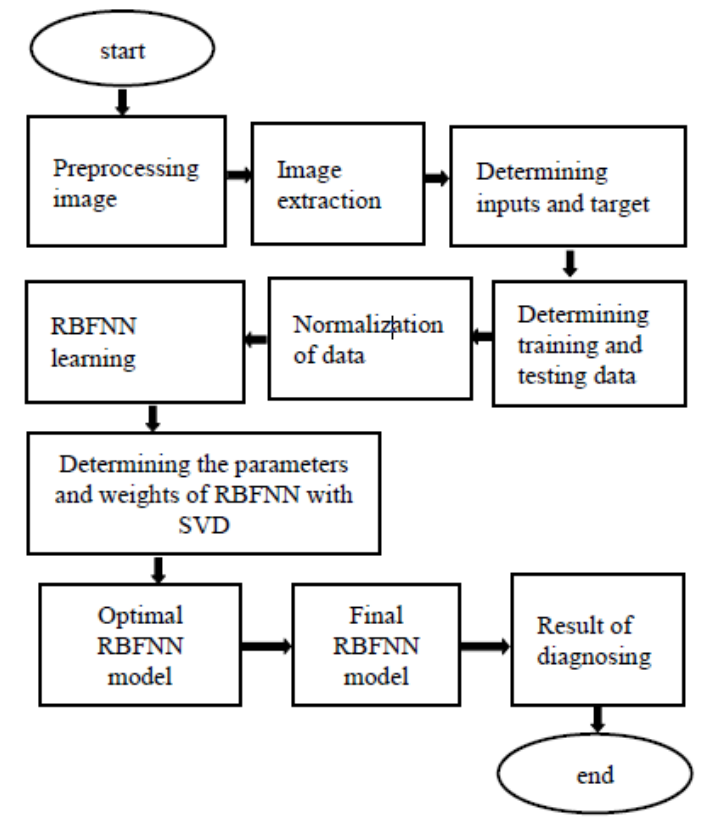

Fig. 2. The diagram of diagnosing brain cancer using RBFNN-SVD.

Estimation of parameters of RBFNN model using SVD is done by the following steps.

1) Preprocessing of data.

2) Image extraction using GLCM.

3) Determining the input and target variables.

4) Dividing of data to training and testing data.

5) Normalizing the data

6) Learning of RBFNN. In this process, the parameters of radial basis function are determined by K-mean clustering. The weights of $\mathrm{NN}$ are determined by SVD method.

7) Determining the optimal weights using SVD.

$$
\boldsymbol{\varphi}_{n \times(q+1)}=U_{n \times n} \Sigma_{n \times(q+1)} V_{(q+1) \times(q+1)}{ }^{T}
$$

where $n$ is the number of training data, $q$ is the number of cluster, $\varphi$ is a matrix of Gaussian activation function for training data, $\boldsymbol{U}$ is a unitary matrix $\Sigma$ is a singular value matrix, $\boldsymbol{V}^{T}=$ unitary matrix

$$
\Sigma=\left[\begin{array}{cccccc}
\sigma_{1} & 0 & 0 & 0 & \cdots & 0 \\
0 & \sigma_{2} & 0 & 0 & \cdots & 0 \\
0 & 0 & \sigma_{3} & \vdots & \cdots & 0 \\
\vdots & \vdots & \vdots & 0 & \ddots & \vdots \\
0 & 0 & 0 & 0 & \sigma_{n} & 0
\end{array}\right]_{n \times(q+1)}
$$

and

$\sigma_{1} \geq \sigma_{2} \geq \sigma_{3} \geq \cdots \geq \sigma_{r} \geq \sigma_{r+1} \geq 0, \sigma_{j}$ is singular value

8) Plotting the singular values of matrix. This step is used to take the nonzero singular values

9) Determining the estimation of $w_{j}$.

$$
U=\left[\begin{array}{llll}
u_{1} & u_{2} & \cdots & u_{n}
\end{array}\right]
$$

where $u_{1}=\left[\begin{array}{c}u_{11} \\ u_{21} \\ \vdots \\ u_{n 1}\end{array}\right], u_{2}=\left[\begin{array}{c}u_{12} \\ u_{22} \\ \vdots \\ u_{n 2}\end{array}\right], u_{N}=\left[\begin{array}{c}u_{1 n} \\ u_{2 n} \\ \vdots \\ u_{n n}\end{array}\right]$,

$$
V^{T}=\left[\begin{array}{llll}
v_{1} & v_{2} & \cdots & v_{(q+1)}
\end{array}\right] \text { where }
$$

$v_{1}=\left[\begin{array}{c}v_{11} \\ v_{21} \\ \vdots \\ v_{(q+1) 1}\end{array}\right], v_{2}=\left[\begin{array}{c}v_{12} \\ v_{22} \\ \vdots \\ v_{(q+1) 2}\end{array}\right], v_{(q+1)}=\left[\begin{array}{c}v_{1(q+1)} \\ v_{2(q+1)} \\ \vdots \\ v_{(q+1)(q+1)}\end{array}\right]$,

Furthermore, the estimation of $w_{j}$ using SVD can be written as (14).

$$
\widehat{w}=\sum_{i=1}^{r} \frac{u_{i}^{T} d}{\sigma_{i}} v_{i}
$$

where $\widehat{w}$ is vector of weights between hidden layer and output layer, $d$ is vector of target on the training data, $u_{i}$ is $i$ th column of matrix $U, \sigma_{i}$ is $i$ th singular value with $\sigma_{i}>$ $0, v_{i}$ is $i$ th column of matrix $V^{T}, r$ is number of nonzero singular values taken.

10) Determining the output of RBFNN-SVD model

The estimation $\widehat{w}$ in (14) is used to estimate $w_{j}$ and we get the RBFNN model as (15).

$$
\hat{y}=\sum_{j=1}^{q} \widehat{w}_{j} \varphi_{j}(x)+w_{0}
$$

\section{RESULTS AND DISCUSSION}

In this section, we apply the proposed method to diagnosis brain cancer as following steps. First, we determine the preprocessing data. In this process, the cropping image of MRI is done. Fig. 3 shows the original image of MRI and the resulted image processing.

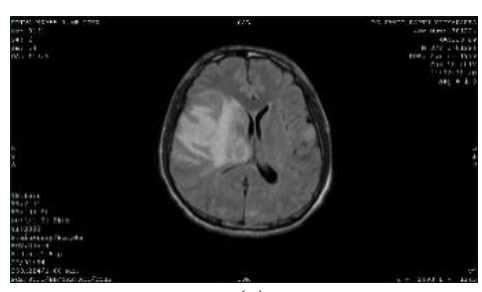

(a)

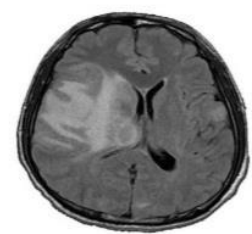

(b)
Fig. 3. (a) original image, (b) result of image preprocessing. 
Then, the image extraction process is done by using GLCM method and there are 14 extractions resulted which are energy, contrast, correlation, sum of squares, inverse difference moment, sum average, sum variance, sum entropy, entropy, difference variance, entropy difference, maximum probability, homogeneity and dissimilarity.

The input variables of the RBFNN are 14 extractions resulted and the targets of RBFNN are normal and abnormal brain. In this study, we divide the data to training and testing data. There are 90 training data and 24 testing data, and then the data are normalized. Then, learning process of RBFNN is done by three steps: the K-mean clustering method to get cluster center and distance, determining the number of hidden neurons by using trial, and then determining the weight of RBFNN by using SVD method. Based on the training data, we set the matrix $\boldsymbol{\varphi}$ using (5). The size of matrix $\boldsymbol{\varphi}$ is $90 \times$ 11 due to the number of training data 90 and the number of cluster 10. Furthermore, the SVD method is applied to the matrix $\varphi$. The singular values in the diagonal matrix from $i$ th row to 11 th row are positive and in other elements are zero. Fig. 4 shows the positive singular values of matrix $\boldsymbol{\varphi}$. Based on Fig. 4, all nonzero singular values are used to determine the weights between hidden layer and output layer (14).

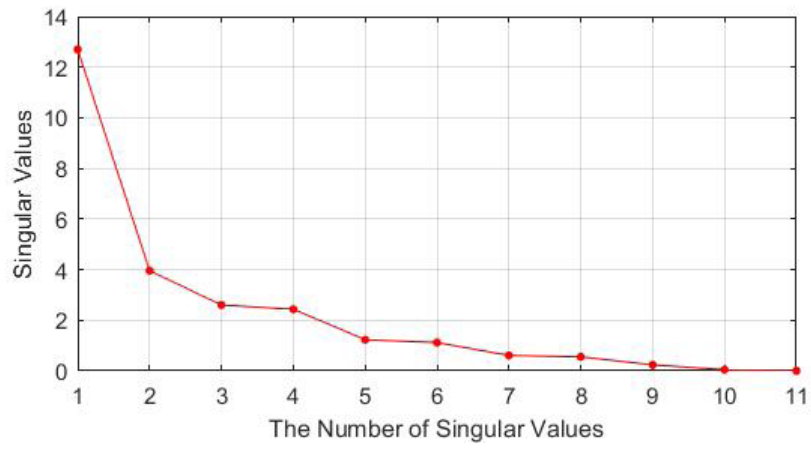

Fig. 4. The singular values of matrix of Gaussian activation function for training data.

Table I shows the model accuracy for some clusters. Based on the Table I, the highest accuracy for training and testing data is achieved at $81.1 \%$ and $91.7 \%$, respectively for ten clusters. Therefore, the optimal number of cluster is ten clusters which are the number of hidden neurons.

\begin{tabular}{ccc} 
TABLE I: COMPARISON OF MODEL ACCURACY FOR SOME CLUSTERS \\
\cline { 2 - 3 } $\begin{array}{c}\text { Number of } \\
\text { cluster }\end{array}$ & $\begin{array}{c}\text { Training data } \\
(\%)\end{array}$ & $\begin{array}{c}\text { Testing data } \\
(\%)\end{array}$ \\
\hline 2 & 57.8 & 66.7 \\
3 & 53.3 & 54.2 \\
4 & 65.6 & 58.3 \\
5 & 64.4 & 54.2 \\
6 & 65.6 & 75 \\
7 & 58.9 & 58.3 \\
8 & 67.8 & 62.5 \\
9 & 70 & 66.7 \\
$10 *$ & 81.1 & 91.7 \\
11 & 78.9 & 87.5 \\
12 & 80 & 87.5 \\
13 & 78.9 & 87.5 \\
14 & 50 & 50 \\
15 & 73.3 & 75 \\
\hline
\end{tabular}

Then, by using (14), we have the weight of RBFNN as follows.

$$
\widehat{\boldsymbol{w}}=\left[\begin{array}{c}
\widehat{w}_{0} \\
\widehat{w}_{1} \\
\widehat{w}_{2} \\
\widehat{w}_{3} \\
\widehat{w}_{4} \\
\widehat{w}_{5} \\
\widehat{w}_{6} \\
\widehat{w}_{7} \\
\widehat{w}_{8} \\
\widehat{w}_{9} \\
\widehat{w}_{10}
\end{array}\right]=\left[\begin{array}{c}
0,56695 \\
1,90672 \\
-2,95405 \\
-3,33099 \\
8,63 \times 10^{-16} \\
0,84061 \\
0,40070 \\
-0,00086 \\
-3,48536 \\
0,557821 \\
1,16185
\end{array}\right]
$$

Based on the weight (16), we have the RBFNN-SVD model as follows.

$$
y=\sum_{j=1}^{10} \widehat{\boldsymbol{w}}_{j} \varphi_{j}(\boldsymbol{x})+w_{0}
$$

In this research, we also estimate the weights of the RBFNN model using back propagation (BP) algorithm. BP algorithm is the most popular learning process in neural networks. The $\mathrm{BP}$ is performed only between hidden layer and output layer. The performance of the RBFNN using SVD and BP

\begin{tabular}{|c|c|c|c|c|}
\hline \multicolumn{5}{|c|}{$\begin{array}{c}\text { TABLE II: COMPARISON OF ACCURACY, SENSITIVITY, AND SPECIFICI } \\
\text { FOR TRAINING AND TESTING DATA USING RBFNN WITH BACK } \\
\text { PROPAGATION AND SVD METHODS }\end{array}$} \\
\hline Method & & $\begin{array}{c}\text { Training } \\
(\%)\end{array}$ & $\begin{array}{c}\text { Testing } \\
(\%)\end{array}$ & $\begin{array}{l}\text { Running } \\
\text { times (s) }\end{array}$ \\
\hline \multirow{3}{*}{ Backpropagation } & Sensitivity & 84.4 & 100 & \multirow{3}{*}{7.087} \\
\hline & Specificity & 77.8 & 83.3 & \\
\hline & Accuracy & 81.1 & 91.7 & \\
\hline \multirow{3}{*}{ SVD } & Sensitivity & 84.4 & 100 & \multirow{3}{*}{3.134} \\
\hline & Specificity & 77.8 & 83.3 & \\
\hline & Accuracy & 81.1 & 91.7 & \\
\hline
\end{tabular}
algorithm is presented in Table II.

Based on the Table II, both models have the same accuracy, sensitivity and specificity for training and testing data. But, the running times of SVD method is less than BP method. This result indicates that SVD is more efficient than BP method.

\section{CONCLUSION}

The RBFNN model can be used for brain cancer diagnosis using MRI image data. The diagnostic steps of brain cancer begin with MRI image processing and image extraction using GLCM. Image extraction results are used as input variables whereas target variables use classification of normal and brain cancer. The data are divided into two sets, namely the training and testing data. Then, the input are normalized. The RBFNN are learned to determine the center and distance of Gaussian activation function using K-means clustering method and to calculate the weight between hidden layer and output layer using back propagation and SVD methods. The performance of the RBFNN model can be considered based on its sensitivity, specificity, and accuracy values. The results show that the sensitivity, specificity, and accuracy of RBFNN diagnoses with backpropagation equals to those of RBFNN with SVD. However, the RBFNN-SVD delivers an advantage in the running speed of the program. In the next study, to increase model accuracy, we will add hidden layer as fuzzification of input. Then, we will apply fuzzy C-mean clustering (FCM) 
method to get optimal cluster center and to determine the optimal number of hidden neurons.

\section{REFERENCES}

[1] HPV Information Center. Humans Papillomavirus and Related Diseases Report INDONESIA. [Online] Available: http://www.hpvcenter.net

[2] Kementrian Kesehatan Republik Indonesia. Pedoman Nasional Pelayanan Dokter Tumor Otak. [Online] Available: http://www.depkes.go.id

[3] A. A. Kathalkar, R. S. Kawitkar, and A. Chopade, "Anficial neural network based brain cancer analysis and classification," International Journal of Computer Application, vol. 66, no. 10, pp. 40-43, 2013.

[4] K. U. Rathod, and Y. D. Kapse, "MATLAB based brain tumor extraction using artificial neural network," International Journal in Recent and Innovation Treand in Comuting and Communication, vol. 4, no. 3, pp. 360-364, 2016.

[5] G. R. Nayak, and T. Verma, "Brain cancer classification using back propagation neural network and principle component analysis," International Journal of Technical Research and Application, vol. 2, no. 4, pp. 26-31, 2014.

[6] P. V. Ramaraju, and S. Baji, "Brain tumour classification, detection and segmentation using digital image processing and probabilistic neural network techniques," International Journal of Emerging Trends in Electrical and Electronics, vol. 10, no. 10, pp. 15-20, 2014.

[7] N. D. Pergad, and K. V. Shingare, "Brain MRI image classification using probabilistic neural network and tumor detection using image segmentation," International Journal of Advanced Research in Computer Engineering and Technology, vol. 4, no. 6, pp. 2946-2951, 2015.

[8] B. Al-Naami, M. A. Mallouh, and E. A. Hafez, "Performance comparison of adaptive neural networks and adaptive neuro-fuzzy inference system in brain cancer classification," Jordan Journal of Mechanical and Industrial Engineering, vol. 8, no. 5, pp. 305-312, 2014.

[9] N. Meghana, and K. R. Rekha, "Artificial neural network based classification of brain tumor from MRI using FCM and bounding method," Internationl Journal of Engineering Research \& Technology, vol. 4, no. 5, pp. 982-985, 2015.

[10] S. Suhag and L. M. Saini, "Automatic brain tumor detection and classification using SVM classifier," in Proc. ISER $2^{\text {nd }}$ International Conference, Singapore, 2015, pp. 55-59.

[11] S. Roy, S. Sadhu, S. K. Bandyopadhyay, D. Bhattacharyya, and T. H. Kim, "Brain tumor classification using adaptive neuro-fuzzy inference system from MRI," International Journal of Bio-Science and Bio-Technology, vol. 8, no. 3, pp. 203-218, 2016.

[12] T. J. Devadas, and R. Ganesan, "Analysis of CT brain images using radial basis function neural network," Defence Science Journal, vol. 62 no. 4, pp. 212-218, 2012.

[13] P. Padmapriya, K. Manikandan, K. Jeyanthi, V. Renuga, and J. Sivaraman, "Detection and classification of brain tumor using radial basis function," Indian Journal of Science and Technology, vol 9, no. 1, pp.1-5, 2016.

[14] P. Jaganathan, N. Rajkumar, and R. Kuppuchamy, "A comparative study of improved F-score with support vector machine and RBF network for breast cancer classification," International Journal of Machine Learning and Computing, vol. 2, no. 6, pp. 741-745, 2012.

[15] S. Hamena and S. Meshoul, "Multi-class classification of gene expression data using deep learning for cancer prediction,' International Journal of Machine Learning and Computing, vol. 8, no. 5, pp. 454-459, 2018.

[16] H. Yoshimura, T. Shimizu, M. Hori, Y. Iwai, and S. Kishid, "EEG signals classification by using an ensemble TPunit neural networks for the diagnosis of epilepsy," International Journal of Machine Learning and Computing, vol. 2, no. 6, pp. 758-761, 2012.
[17] A. M. Abadi, D. U. Wutsqa, and L. R. Pamungkas, "Detection of lung cancer using radiograph images enhancement and radial basis function classifier," in Proc. 2017 10th International Congress on Image and Signal Processing, BioMedical Engineering and Informatics, CISP-BMEI, October 2017.

[18] D. B. Andrew, Radial Basis Function. Handbook of Neural Network, Signal Processing, 2002.

[19] R. A. Johnson and D. W. Wichern, Applied Multivariate Statistical Analysis, 6th ed., Upper Saddle River, New Jersey: Pearson, 2007.

[20] J. T. Scheick, Linear Algebra with Applications, Singapore: McGraw-Hill, 1997.

[21] W. Zhu, N. Zeng, and N. Wang, "Sensitivity, specificity, accuracy, associated confidence interval and ROC analysis with practical SAS® implementations," Health Care and Life Sciences, pp. 1-9, 2010.

[22] D. Gadkari, "Image quality analysis using GLCM," Thesis, University of Central Florida, 2004.

[23] R.M. Haralick, K. Shanmugam, and I. Dinstein, "Textural features for image classification," IEEE Transactions on Systems, Man and Cybernetics, vol. SMC-3, no. 6, pp. 610-621, 1973.

[24] D. U. Wutsqa and M. Marwah, "Median filter noise reduction of image and backpropagation neural network model for cervical cancer classification," Journal of Physics: Conference Series, vol. 855, 2017.

[25] D. U. Wutsqa and P. S. Rahmada, "Point operation to enhance the performance of fuzzy neural network model for breast cancer classification," Journal of Engineering and Applied Science, vol. 12, no. 17 , pp. $4405-441,2017$

[26] D. U. Wutsqa and C. L. R. Mandadara," Lung cancer classification using radial basis function neural network model with point operation,' in Proc. 10th International Congress on Image and Signal Processing, BioMedical Engineering and Informatics, CISP-BMEI, October 2017.

[27] O. B. Abouelatta, "Classification of copper alloys microstructure using image processing and neural network," Journal of America Science, vol. 9 , pp. 213-223, 2013

[28] A. B. Girisha, M. C. Chandrashekhar, and M. Z. Kurian, "Texture feature extraction of video frames using GLCM," International Journal of Engineering Trends and Technology, vol. 4, pp. 2718-2721, 2013.

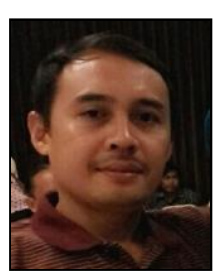

Agus Maman Abadi received his Dr. degree from Gadjah Mada University, Indonesia in 2010. Presently, he is an associate professor of Mathematics department, Yogyakarta State University, Indonesia. His current research interests include fuzzy time series model, fuzzy systems, neural network model, image processing, and fuzzy decision making.

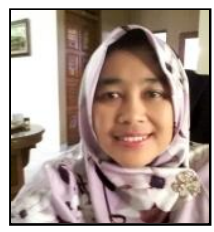

Dhoriva Urwatul Wustqa received her Dr. degree from Gadjah Mada University, Indonesia in 2008. Presently, she is an associate professor of Mathematics Department, Yogyakarta State University, Indonesia. Her current research interests include fuzzy time series model, neural network model, and image processing.

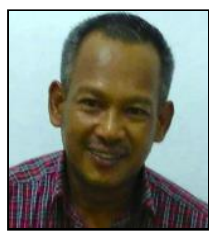

Nurhayadi received his Dr. degree from Gadjah Mada University, Indonesia, in 2014. Presently, he is an associate professor of Mathematics Education Department, Tadulako University, Indonesia. His current research interests include fuzzy time series model, fuzzy systems, and neural network model. 